\title{
¿ES EFECTIVA LA CONSEJERÍA PARA AUMENTAR EL CONSUMO DE FRUTAS Y VERDURAS Y DISMINUIR EL RIESGO CARDIOVASCULAR EN PREVENCIÓN SECUNDARIA? UNA REVISIÓN.
}

\author{
IS COUNSELING AN EFFECTIVE STRATEGY TO INCREASE FRUITS AND \\ VEGETABLES CONSUMPTION AND DECREASE THE CARDIOVASCULAR RISK \\ IN SECONDARY PREVENTION? A REVIEW.
}

\author{
Álvaro Castillo C.(1), Claudia Smith G. (2), Sandra Hirsch B. (1), Alex Brito O. (1) \\ (1) Instituto de Nutrición y Tecnología de los Alimentos (INTA), Universidad de Chile \\ (2) Departamento de Medicina Familiar, Pontificia Universidad Católica de Chile
}

\begin{abstract}
Introduction: Consumption of fruits and vegetables, due to their high fiber and antioxidant content, has been associated with an improvement in some biological parameters that modify cardiovascular risk, such as total cholesterol, LDL cholesterol and blood pressure. One of the most investigated strategies to increase the consumption of vegetables is counselling. Method: We searched the MEDLINE database and other review references. Results: 42 abstracts were reviewed (36 from MEDLINE and 6 of United States Preventive Service Task Force Review) and 30 were selected. Of the 30, 8 match with the inclusion criteria. We found solid epidemiological evidence that showed the association between the intake of fruits and vegetables and cardiovascular risk factors. We did not find enough evidence to demonstrate that counseling is effective in modifying the risk profile in secondary prevention. Conclusion: counseling produces modest changes in the diet and the risk profile in healthy patients, but there is still a need for more information about its effectiveness in long term changes in patients with high cardiovascular risk.
\end{abstract}

Key words: fruit and vegetables, cardiovascular risk, counseling.

Este trabajo fue recibido el 25 de Enero de 2008 y aceptado para ser publicado el 18 de Abril de 2008

\section{INTRODUCCIÓN}

Las enfermedades cardiovasculares (ECV) han aumentado dramáticamente en las últimas décadas y constituyen la principal causa de muerte en Chile (1). La mortalidad por ECV en el año 1970 representaba el 15,2\% del total de las muertes, mientras que en el 2005 llegó al 28,2\%, de las cuales un 9,2\% corresponden a infarto al miocardio (IAM) y un 9\% a accidente vascular encefálico (AVE). 
Según datos de la $1^{\text {a }}$ Encuesta Nacional de Salud (ENS) (2) realizada el año 2003, la prevalencia de riesgo cardiovascular (RVC) alto o muy alto, definido como la presencia de $2 \mathrm{o}$ más factores de riesgo, tener diabetes o enfermedad ateroesclerótica, alcanza a más del $50 \%$ de la población general, cifra que aumenta al $80 \%$ en los mayores de 65 años.

La asociación entre los estilos de vida y el RCV ha sido ampliamente estudiada. Dentro de éstos, el hábito tabáquico (3-6), consumo excesivo de alcohol (7-9) la inactividad física (10-13) y una dieta inadecuada se han asociado a un aumento del RCV. Dentro del ámbito nutricional, existe evidencia que asocia el aumento del riesgo al consumo de grasas saturadas (14) y trans $(15-17)$ y la ingesta elevada de sodio $(18,19)$. Pero así como se han descrito factores de riesgo también se han descrito algunos factores protectores como el consuno de ácidos grasos omega 3 de cadena larga EPA y DHA (14), y el consumo de frutas (F) y verduras (V) de manera regular (20).

Dentro de los elementos que explican la asociación inversa entre RCV y consumo de F y V se han mencionado el alto contenido de fibra y antioxidantes, que serían responsables de la disminución del colesterol total, colesterol LDL y de la presión arterial.

En este sentido, el aumento del consumo de F y V se ha planteado como una estrategia que ayudaría a mejorar el perfil de RCV de la población, siendo la consejería una de las herramientas más utilizadas a nivel individual.

Para los efectos de este trabajo, entenderemos consejería como consejo asistido (21) por un profesional de salud o como traducción de los términos counseling, advice o nutritional education

En esta revisiónanalizamos la evidencia actual que investiga el consumo de $\mathrm{F}$ y $\mathrm{V}$ y la efectividad de la consejería como estrategia de salud pública para mejorar el perfil de RCV en prevención secundaria.

\section{MÉTODOS}

\section{Búsqueda de la información}

La búsqueda de la información pretende responder tres preguntas cuya progresión lógica nos permitirían abarcar el tema de manera general. La primera pregunta fue ¿cuál es el impacto de una dieta rica en $\mathrm{F}$ y $\mathrm{V}$ en el RCV? La segunda fue ¿El aumento del consumo de $\mathrm{F}$ y $\mathrm{V}$ mejoran el perfil de riesgo en pacientes con RCV? Y la tercera fue ¿La consejería logra aumentar el consumo de $\mathrm{F}$ y $\mathrm{V}$ y disminuir el riesgo en pacientes con RCV? A partir de estas tres preguntas nos propusimos hacer una búsqueda en MEDLINE, y en referencias de otras revisiones.

Los descriptores utilizados fueron: "diet", "fruit", "vegetables", "cardiovascular disease", "counseling" y "nutritional education". Se utilizaron los integradores AND y OR.

\section{Criterio de inclusión}

Los criterios de inclusión para la pregunta número uno fueron: Ensayos Clínicos Aleatorizados y Controlados (RCT), Estudios de caso y control(CCT), estudios prospectivos, retrospectivos, revisiones sistemáticas, meta análisis; artículos de 1997 en adelante; edad $\geq$ a 18; consumo cuantificado de frutas y verduras.

Para la pregunta número dos fueron: RCT, CCT, revisión sistemática, meta análisis de estudios aleatorizados; artículos de 1997 en adelante; edad $\geq$ a 18; pacientes con uno o más 
factores de RCV; consumo cuantificado de frutas y verduras; asociación entre cambio en el consumo de frutas y verduras y riesgo cardiovascular; grupo control

Los criterios para la pregunta número tres fueron: RCT; artículos de 1997 en adelante; edad $\geq$ a 18; pacientes con uno o más factores de RCV; consumo cuantificado de frutas y verduras; consejería realizada por un profesional de la salud; asociación entre cambio en el consumo de frutas y verduras y riesgo cardiovascular; grupo control

\section{RESULTADOS}

Se revisaron 42 resúmenes, 36 de Pub Med y 6 de otra revisión del tema del United States Preventive Service Task Force, (USPSTF) de los cuales se seleccionaron 30. De estos 30, 8 cumplieron con los criterios de inclusión ya mencionados.

\section{¿Cuál es el impacto de una dieta rica en $F$ y $\mathrm{V}$ en el RCV?}

La información que arrojó la búsqueda en la pregunta uno corresponde a evidencia epidemiológica de estudios de cohorte y meta-análisis que analizaron la asociación entre el consumo de F y V y RCV.

En el primer estudio se examinó en una cohorte de mujeres (22) la hipótesis de que el aumento en le consumo de F y V reduciría el RCV. Se midió el consumo de F y V en 39.876 mujeres sin historia previa de ECV o cáncer con un cuestionario de consumo. El seguimiento fue de 5 años en promedio y los resultados medidos fueron la incidencia de IAM no fatal, AVE, angioplastía coronaria, bypass arterial o muerte por ECV. En este estudio se encontró una asociación inversa y estadísticamente significativa $(\mathrm{p}=0,01)$ entre los extremos de consumo de $\mathrm{F}$ y $\mathrm{V}$ en quintiles $(2,6$ - 10,2 porciones/día) y el riesgo de ECV ajustado por edad, tipo de tratamiento y hábito tabáquico (Riesgo Relativo (RR) 1 - 0,68). Sin embargo, al ajustar por otras variables de RCV también hubo una asociación inversa pero no significativa. $\mathrm{Al}$ excluir el autoreporte de diabetes, hipertensión, colesterol elevado al inicio, el RR del $5^{\circ}$ quintil fue de $0,45(\mathrm{p}=0,09)$. También, el mayor consumo de $\mathrm{F}$ y $\mathrm{V}$ se asoció de manera inversa con IAM con un RR del $5^{\circ}$ quintil de 0,62 (P:0,07). Como conclusión, en este estudio se sugiere que un aumento en el consumo de $\mathrm{F}$ y $\mathrm{V}$ puede tener un efecto protector ante ECV y apoya las actuales recomendaciones de aumentar su consumo.

En otro estudio prospectivo (23) se examinó la asociación entre la fuente de fibra dietaria y el riesgo de ECV en una cohorte de hombres y mujeres. Se analizaron separadamente al grupo de mujeres $(n=3429)$ y de hombres $(n=2532)$ en un modelo de regresión logística donde los quintiles de consumo de fibra fueron la variable independiente. Aquí se vio que la fibra proveniente de vegetales se asocia con una disminución en la presión arterial y la concentraciones de homocisteína, que la fibra proveniente de fruta se relaciona con una menor relación cintura/cadera y presión arterial y que la fibra proveniente de frutos secos y semillas se asocia con un menor índice de masa corporal (IMC), relación cintura/cadera y concentraciones de glucosa en ayuno. Los autores concluyen que la fibra dietaria se asocia inversamente $(\mathrm{p}<0.05)$ con varios factores de $\mathrm{RCV}$, y que se recomienda aumentar su consumo.

En la cohorte de las enfermeras $(\mathrm{n}=84.251)$ y en la cohorte los profesionales de salud ( $\mathrm{n}$ =42.148) (24) se estudió la asociación entre el consumo de $\mathrm{F}$ y V y el riesgo de enfermedad coronaria (EC). Las poblaciones fueron sanas al inicio y se realizó un seguimiento promedio de 14 años. Las variables dependientes fueron IAM no fatal y muerte por EC, se reportaron 
1127 casos en mujeres y 1063 casos en hombres. El consumo de F y V se registró través de cuestionarios de frecuencia de consumo. EL RR de EC en el quintil más alto de consumo fue de 0.80 (95\% Intervalo de Confianza (IC) 0,69 a 0,93) al ser comparado con el quintil más bajo. Según este estudio, el riesgo de EC disminuía en un $4 \%$ por cada porción de $\mathrm{F}$ y $\mathrm{V}$ al día (RR 0,96 con un 95\% IC del 0,94 a 0,99), siendo las frutas ricas en vitaminas $\mathrm{C}$ y las verduras de hoja verdes las con mayor efecto protector. Los autores conc luyen que el consumo de $\mathrm{F}$ y $\mathrm{V}$, parecen ser un factor protector de EC.

En un meta-análisis (25) reciente de estudios de cohorte se buscaron estudios prospectivos que reportaran el RR o hazard ratio (HR) con IC del 95\% de AVE relacionado con el consumo de F y V a través de cuestionarios de frecuencia de consumo validados. Se seleccionaron 8 estudios a partir de 9 cohortes independientes con una muestra total de 257.551 personas y 4917 eventos AVE con un seguimiento promedio de 13 años. Al comparar el grupo que reportó un consumo $<3$ porciones/día de $\mathrm{F}$ y $\mathrm{V}$ con los que comían entre 3-5 y $>5$ p/d, el pool de RR fue de 0.89 (95\% IC 0.83-0.97) y 0.74 (95\% IC 0.69-0.79) respectivamente.

La conclusión de este meta análisis es que existe una asociación entre el mayor consumo de frutas y verduras y una reducción del riesgo de AVE, y constituye un aporte a la actual recomendación de comer al menos 5 porciones al día.

En otro meta-análisis (26), buscaron estudios de cohorte que reportaran RR para cualquier tipo de AVE y que cuantificaran el consumo de frutas y verduras con cuestionarios validados. Se seleccionaron 7 estudios con una muestra total de 94.513 hombres, 141.536 mujeres y se registraron 955 eventos AVE. No hubo heterogeneidad entre los estudios por lo que el análisis integrado arrojo un disminución del RR en un 11\% (95\% IC 0,85 a 0,93) por cada porción adicional de $\mathrm{F}$, de un $5 \%$ (IC 95\% 0,92 a 0,97) por cada porción adicional de $\mathrm{F}$ y $\mathrm{V}$, sin embargo, no se encontró un efecto protector en el consumo de $\mathrm{V}$ de forma independiente. Los autores concluyen que el consumo de $\mathrm{F}$ y de $\mathrm{F}$ y $\mathrm{V}$ disminuyen el riesgo de AVE.

También encontramos un meta- análisis de estudios de cohorte del mismo autor anterior (27) pero que analizó la asociación entre el consumo de F y V con EC o mortalidad por esta causa. Aquí se seleccionaron 9 estudios que reportaran RR para EC o mortalidad y que mostraran consumo cuantificado de F y V. La muestra total fue de 91.379 hombres, 129.701 mujeres y se reportaron 5.007 eventos de EC. Aquí encontraron que el Pool RR para el consumo de F y V fue de 0.96 (95\% IC 0.93-0.99) al aumentar en una porción el consumo, de 0.93 (95\% IC 0.89-0.96) al aumentar en una porción el consumo de F, sin embargo, no se pudo concluir nada respecto al consumo de $\mathrm{V}$ de forma independiente, dado de el test de heterogeneidad para esta variable fue significativo. En este meta-análisis se concluye que existe una relación inversa entre el consumo de $\mathrm{F}$ y de $\mathrm{F}$ y $\mathrm{V}$ con el riesgo de $\mathrm{EC}$, pero que sigue $\mathrm{n}$ pendiente los mecanismos que explican esta asociación.

\section{¿El aumento del consumo de $\mathrm{F}$ y $\mathrm{V}$ mejoran el perfil de riesgo en pacientes con RCV?}

Cuando realizamos la búsqueda para responder la pregunta dos, el volumen de la información fue bastante menor que para la primera, aunque si encontramos un estudio que investigó el efecto preventivo del pomelo hibrido (sweeties fruit) en pacientes hipercolesterolémicos con EC (28). Aquí se seleccionaron 66 pacientes voluntarios entre 47 y 68 años con bypass coronario, los que fueron asignados aleatoriamente a 3 grupos, uno 
control, uno que recibió una fruta al día y otro que recibió 2. Los pacientes seleccionados no tomaron hipolipemiantes durante los 30 días que duró la investigación. Aquí hallaron que los pacientes que recibieron 1 y 2 frutas bajaron en un $-8.7 \%$ (NS) y un $-16.1 \%(<0.01)$ el colesterol total y en un $-1,6 \%(\mathrm{p}<0.01)$, en un $-21,2 \%(\mathrm{p}<0.0005)$ el colesterol LDL, y en un $11,5 \%$ (NS) y un $24,7 \%$ ( $<<0.0005)$ los triglicéridos en relación al grupo control, sin embargo, no tuvieron cambios significativos en el colesterol HDL. En este estudio se concluyó que la dieta suplementada con pomelo híbrido influencia positivamente el metabolismo de los lípidos y la capacidad antioxidante y que puede ser benéfico en la prevención de arterioesclerosis, especialme nte en pacientes hipercolesterolémicos

\section{¿La consejería logra aumentar el consumo de $F$ y $V$ y disminuir el riesgo en pacientes con RCV?}

$\mathrm{Al}$ analizar el efecto de al consejería para aumentar el consumo de $\mathrm{F}$ y $\mathrm{V}$ en pacientes con RCV, encontramos sólo un estudio que cumplió con los criterios de inclusión (29). En este estudio se vio la asociación entre aumento del consumo de $\mathrm{F}$ y $\mathrm{V}$ con consejo simple (advise) y una reducción de la mortalidad después de IAM. La muestra fue de 11.323 personas con IAM que fueron asignadas aleatoriamente a una dieta mediterránea (pescado, frutas, verduras crudas y cocidas y aceite de oliva) y a una dieta control, con un seguimiento promedio de 6,5 años. Aquí se observó que comparado con aquellos que no consumen fruta, el Odds Ratio (OR) de los que comen $>1 \mathrm{p} / \mathrm{d}$ ajustando por sexo, edad, fumador, variables bioquímicas y las otras variables dietarias, fue de 0.73 (95\% IC 0.54-0.98). Comparado con aquellos que no cons umen verduras crudas, el OR ajus tado de los que comen 2-3 p/semana, 1 p/día y >1 p/día fue de $0.83(0.70$ - 0.98), $0.67(0.56-0.79)$ y 0.65 (0.51 - 0.84) respectivamente. Y comparado con aquellos que no consumen verduras cocidas, el OR ajuntado de los que comen $>1 \mathrm{p} /$ día fue $0.70(0.50-0.99)$. Aquí se concluyó que el consejo simple ayuda a aumentar el consumo de $\mathrm{F}$ y $\mathrm{V}$ en pacientes con IAM y lleva a una reducción sustancial del riesgo de muerte temprana, por lo que se recomienda que los clínicos incentiven el consumo.

\section{DISCUSIÓN}

En esta revisión hemos visto que la evidencia epidemiológica que apoya el consumo de F y V como un elemento protector de las ECV es sólida y conc uerda con aquella que dice que los vegetarianos tienen una menor morbimortalidad por estas causas (30), lo cual sustentaría la idea de incentivar un consumo más ele vado. Sin embargo, aun no está del todo claro la asociación que existe entre la recomendación de aumentar el consumo de $\mathrm{F}$ y $\mathrm{V}$ y el impacto de esa recomendación en el perfil de RCV, en especial en prevención secundaria.

En esta revisión nos encontramos con que el número de artículos que cumplieran con los criterios de inclusión para la pregunta 2 y 3 fue bajo, ya que de todos los estudios que arrojó la búsqueda sólo los dos comentados cumplieron con los criterios de inclusión. En general, el mayor volumen de información en prevención secundaria estaba referido a la consejería y dieta mediterránea (31-33), donde no se evaluaba el efecto de $\mathrm{F}$ y V en los factores de RCV de forma independiente. Otro aspecto que limitó la búsqueda, fue el que decía relación con ha población en la cual nos centramos, ya que en gran parte de los trabajo el foco eran los pacientes sanos y no aquellos con RCV. Pensamos que las limitaciones en la búsqueda estuvieron dadas en primer lugar, a que existe poca investigación del tema con muestras con 
RCV; y en segundo lugar, a que la búsqueda puede haber estado limitada al no incluir otros buscadores que pudiera haber complementado lo encontrado en Medline y la revisión sistemática del USPSTF.

En cuanto a esa revisión sistemática $(34,35)$, que incluyó artículos entre el año 1966 y 2001, se analizó la efectividad de la consejería para modificar la dieta en pacientes sanos y se concluyó que la consejería produce cambios moderados en el consumo de F y V $(0,2$ a 0,9 pociones por día) y que el cambio es mayor a medida que aumenta el número de factores de riesgo de los paciente, lo que apoyaría la hipótesis de que la consejería podría tener resultados significativos en mejorar el perfil de riesgo en prevención secundaria. De todas maneras la recomendación respecto al uso de la consejería para mejorar la dieta es "I", es decir, con evidencia insuficiente para recomendar la práctica clínica.

En otra revisión sistemática publicada en Octubre del 2007 en la Cochrane Database (36), se evaluó si la consejería para aumentar el consumo de $\mathrm{F}$ y $\mathrm{V}$ mejora el perfil de riesgo cardiovascular en adultos sanos. Los autores vieron que hubo cambios modestos aunque significativos en algunas variables dietarias, como el aumento del consumo de F y V(1.25 porciones/día), consumo de fibra y disminución del consumo de grasas totales y saturadas. Además se observó una disminución significativa de la presión sistólica y diastólica, el colesterol total y el colesterol LDL; no se modificó así el colesterol HDL y los triglicéridos. Con estos resultados, los autores concluyen que la consejería para aumentar el consumo de frutas y verduras produciría efectos modestos en la dieta y en los factores de RCV, pero que se necesita más evidencia sobre sus efectos a largo plazo.

En Chile, el consumo de $\mathrm{F}$ y $\mathrm{V}$ fue estudiado por Olivares y cols, quienes vieron que los niños de entre 8 y 9 años de edad consumen entre 2 y 3 pociones de $\mathrm{F}$ y $\mathrm{V}$ al día en promedio (164-229g/día) $(37,38)$ siendo los grupos más pobres los que menos consumen. La Encuesta de Calidad de Vida y Salud del año 2006 (39) mostró que solamente el 52\% de la población adulta reconoce comer verduras todos los días y el $47 \%$ frutas, siendo nuevamente las poblaciones más pobres, los grupos de menor edad y los hombres los que menos consumen. En relación a esta realidad y a la evidencia epidemiológica con que se cuenta, es que existe un programa nacional orientado a fomentar e consumo diario de 5 porciones de $\mathrm{F}$ y $\mathrm{V}$. Esta recomendación está basada en la recomendación hecha por la OMS y tiene por objetivo modificar el patrón de consumo de la población. La campaña "5 al día" es algo que ha sido instaurado en varios países entre los que se encuentra Estado Unidos y España, campaña que ha ido más allá en países como Canadá donde la campaña habla de 5 a 10 al día.

En cuanto a la consejería, xtualmente existen esfuerzos por introducirla en la práctica clínica como una herramienta que ayude a mejorar el estado de salud de los pacientes y la adherencia a los tratamientos indicados. Si bien aun existe discusión respecto a su efectividad y a cuál es el mejor modelo conceptual, resulta coherente investigar el tema bajo la hipótesis de que la consejería para aumentar el consumo de $\mathrm{F}$ y V en pacientes con RCV, sería de ayuda en la prevención y tratamiento de las enfermedades asociadas, en especial en países con el perfil de consumo y epidemiológico de Chile, donde sabemos que el consumo de $\mathrm{F}$ y $\mathrm{V}$ es bajo y la prevalencia de ECV es alta. 


\section{CONCLUSIONES}

La evidencia epidemiológica actual apoya la hipótesis de que el consumo de $\mathrm{F}$ y $\mathrm{V}$ tiene un efecto protector en el RCV, aunque el efecto de las $\mathrm{V}$ al ser analizados de forma independiente no es concluyente.

La consejería parece tener un efecto modesto en el aumento del consumo de $\mathrm{F}$ y $\mathrm{V}$ y en el perfil de riesgo CV en pacientes sanos. Sin embargo, existe información insuficiente acerca de su impacto en portadores de ECV, por lo que se hace necesario investigar el efecto que pudiera tener en prevención secundaria, tanto en el mediano como en el largo plazo.

\section{RESUMEN}

Introducción: El consumo de frutas y verduras, dado su alto contenido de fibra y antioxidantes, se ha asociado a una mejoría de algunos de los parámetros biológicos que modifican el riesgo cardiovascular, como el colesterol total, colesterol LDL y la presión arterial. Dentro de las estrategias dirigidas a aumentar la ingesta de vegetales, la consejería es una de las más estudiadas. Métodos: se realizó una búsqueda en la base de datos MEDLINE, y en referencias de otras revisiones. Resultados: se revisaron 42 resúmenes (36 de MEDLINE y 6 de la revisión del United States Preventive Service Task Force) de los cuales se seleccionaron 30. De estos 30, 8 cumplieron con los criterios de inclusión. Se encontró evidencia epidemiológica sólida que asoció el consumo de frutas y verduras a una disminución del riesgo cardiovascular (RCV). No encontramos evidencia suficiente respecto a la efectividad de la consejería para modificar el perfil de riesgo en prevención secundaria. Conclusiones: la consejería produce cambios modestos en la dieta y el perfil de riesgo en pacientes sanos, sin embargo se necesita más información respecto su efectividad en pacientes con enfermedad CV y a sus efectos a largo plazo.

Palabras claves: frutas y verduras, riesgo cardiovascular, consejería.

Dirigir la correspondencia a:

Profesor

Alvaro Castillo C.

INTA, Universidad de Chile

Av. El Líbano 5524

Casilla 138-11

Santiago, Chile

Fono: 92350944

E-mail: acastillo@inta.cl 


\section{BIBLIOGRAFÍA}

1. Ministerio de Salud, Departamento de Estadśticas e Información de Salud (DEIS)

2. Encuesta Nacional de Salud 2003

3. Critchley J, Capewell S. Smoking cessation for the secondary prevention of coronary heart disease. Cochrane Database of Systematic Reviews 2003, Issue 4.

4. Woodward M, Lam TH, Barzi F, Patel A, Gu D, Rodgers A, et al; Asia Pacific Cohort Studies Collaboration Smoking, quitting, and the risk of cardiovascular disease among women and men in the Asia-Pacific region. Int J Epidemiol 2005;34(5):1036-45

5. Kaur S, Cohen A, Dolor R, Coffman CJ, Bastian LA. The impact of environmental tobacco smoke on women's risk of dying from heart disease: a meta-analysis. $\underline{\mathrm{J}}$ Womens Health (Larchmt) 2004;13(8):888-97.

6. Rice VH, Stead LF. Nursing interventions for smoking cessation. Cochrane Database of Systematic Reviews 2004, Issue 1.

7. K Srinath Reddy, Martijn B Katan. Diet, nutrition and the prevention of hypertension and cardiovascular diseases. Public Health Nutr 2004;7(1A):167-86.

8. Chrysohoou C, Panagiotakos DB, Pitsavos C, Skoumas J, Toutouza M, Papaioannou I, et al. Effects of chronic alcohol consumption on lipid levels, inflammatory and haemostatic factors in the general population: the 'ATTICA' Study. Eur J Cardiovasc Prev Rehabil 2003 ;10(5):355-61.

9. Iso H, Kitamura A, Shimamoto T, Sankai T, Naito Y, Sato S, el at. Alcohol intake and the risk of cardiovascular disease in middle-aged Japanese men. Stroke 1995;26(5):767-73.

10. Wannamethee SG, Shaper AG, Walker M. Changes in physical activity, mortality, and incidence of coronary heart disease in older men. Lancet 1998;351(9116):1603-8

11. Barengo NC, $\underline{\mathrm{Hu} G}, \underline{\text { Lakka TA}}, \underline{\text { Pekkarinen H}}$, Nissinen A, Tuomilehto J. Low physical activity as a predictor for total and cardiovascular disease mortality in middleaged men and women in Finland. Eur Heart J 2004;25(24):2204-11.

12. Wannamethee SG, Shaper AG, Walker M. Physical activity and mortality in older men with diagnosed coronary heart disease. Circulation 2000;102 (12):1358-63.

13. Wannamethee SG, Shaper AG. Physical activity in the prevention of cardiovascular disease: an epidemiological perspective. Sports Med 2001;31(2):101-14. 
14. Frank B. Hu, JoAnn E. Manson, and Walter C. Willett, Types of Dietary Fat and Risk of Coronary Heart Disease: A Critical Review. J Am College Nutr 2001;20, 1, 5-19.

15. Mozaffarian D. Katan M. Ascherio, A. Stampfer, M. and Willett, W. Trans Fatty Acids and Cardiovascular Disease. N Engl J Med 2006;354:1601-13.

16. Lichtenstein AH, Ausman LM, Jalbert, SM, Schaefer EJ. Effects of different forms of dietary hydrogenated fats on serum lipoprotein cholesterol levels. N Engl J Med 1999;340:1933-40.

17. Oomen CM, Ocke MC, Feskens EJ, van Erp-Baart MA, Kok FJ, Kromhout D. Association between trans fatty acid intake and 10-year risk of coronary heart disease in the Zutphen Elderly Study: a prospective population-based study. Lancet 2001; $357: 746-51$.

18. Sacks FM. Svetkey LP. Vollmer WM. Effects on blood pressure of reduced dietary sodium and the dietary approaches to stop hypertension (DASH) diet. DASH-Sodium Collaborative Research Group. N Engl J Med 2001;344 3 -10

19. FJ He, GA MacGregor. Effect of longer-term modest salt reduction on blood pressure. Cochrane Database of Systematic Reviews 2007 Issue 4

20. Andrew R. Ness and John W. Powles. Fruit and Vegetables, and Cardiovascular Disease: A Review. Internat J Epidemiol 1997;26:1-13.

21. J.L. Bimbela. El Counselling: una tecnología para el bienestar del profesional. ANALES Sis San Navarra 2001; 24 (Supl. 2):33-42.

22. Liu, S. Manson, JA. Lee, IM. Cole, S. Hennekens, C. Willett, W. et al. Fruit and vegetable intake and risk of cardiovascular disease: the Women's Health Study. Am J Clin Nutr 2000;72:922-8.

23. Lairon, D. Arnault N. Bertrais, S. Planells, R. Clero, E. Hercberg, S. et al. Dietary fiber intake and risk factors for cardiovascular disease in French adults. Am J C lin Nutr 2005;82:1185-94.

24. Joshipura, K. Hu, F. Manson, JA. Stampfer, M. Rimm, E. Speizer, F. et al. The Effect of Fruit and Vegetable Intake on Risk for Coronary Heart Disease. Ann Intern Med 2001:134:1106-1114.

25. Feng J. He, Caryl A. Nowson, Graham A. MacGregor. Fruit and vegetable consumption and stroke: meta-analysis of cohort studies. Lancet 2006; 367: 320-26

26. Dauchet, L. Amouyel, P. and Dallongeville, J. Fruit and vegetable consumption and risk of stroke: A meta-analysis of cohort studies. Neurology 2005;65:1193-1197. 
27. Dauchet, L. Amouyel, P. and Dallongeville, J. Hercberg, S. and Dallongeville, J. Fruit and Vegetable Consumption and Risk of Coronary Heart Disease: A Meta-Analysis of Cohort Studies. J Nutr 136: 2588-2593, 2006.

28. Gorinstein, S. Caspi, A. Libman, I. Katrich, E Tzvi Lerner, H. and Trakhtenberg. S. Preventive effects of diets supplemented with sweetie fruits in hypercholesterolemic patients suffering from coronary artery disease. Preventive Med 2004;(38), 841-847.

29. Barzi, F Woodward, M Marfisi, RM Tavazzi, L Valagussa F and Marchioli R. On behalf of GISSI-Prevenzione Investigators Mediterranean diet and all-causes mortality after myocardial infarction: results from the GISSFPrevenzione trial. European J Clin Nutr 2003; 57, 604-611.

30. Key, T. Fraser, G. Thorogood, M. Appleby, PN. Beral, V. Reeves, G. Mortality in vegetarians and nonvegetarians: detailed findings from a collaborative analysis of 5 prospective studies. Am J Clin Nutr 1999;70(suppl):516S-24S.

31. Estruch, R. Martinez-González, MA. Corella, D. et al. Effects of a MediterraneanStyle Diet on Cardiovascular Risk Factors: A Randomized Trial. Ann Intern Med 2006;145:1-11.

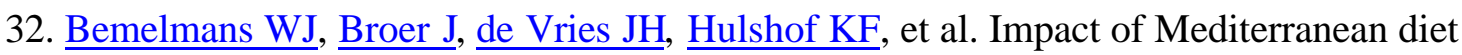
education versus posted leaflet on dietary habits and serum cholesterol in a high risk population for cardiovascular disease. Public Health Nutr 2000;3(3):273-83.

33. de Lorgeril M, Salen P, Martin JL, Monjaud I, Delaye J, Mamelle N. Mediterranean diet, traditional risk factors, and the rate of cardiovascular complications after myocardial infarction: final report of the Lyon Diet Heart Study. Circulation 1999;99(6):779-85

34. Ammerman, A. Pignone, M. Fernandez, L. Lohr, K. Driscoll-Jacobs A. Nester, C. et al. Counseling to Promote a Healthy Diet Systematic Evidence Re view U.S.

Preventive Services Task Force 2002 (18).

35. U.S. Preventive Services Task Force. Behavioral Counseling in Primary Care to Promote a Healthy Diet: Recommendations and Rationale. Am J Prev Med 2003; 24(1):93-100.

36. Brunner EJ, Rees K,Ward K, BurkeM, ThorogoodM. Dietary advice for reducing cardiovascular risk. Cochrane Database of Systematic Reviews 2007, Issue 4.

37. Olivares C, Sonia, Bustos Z, Nelly, Lera M, Zelada, ME. Estado nutricional, consumo de alimentos y actividad física en escolares mujeres de diferente nivel socioeconómico de Santiago de Chile. Rev Méd Chile 2007;135(1)71-78. 
38. Olivares C, Sonia y Bustos Z, Nelly. Consumo de verduras y frutas en grupos específicos de consumidores chilenos: elementos a considerar en su promoción. Rev Chil Nutr 2006;33; (supl.1)260-264

39. II Encuesta de Calidad de Vida y Salud Chile,. Ministerio de Salud, Subsecretaría de Salud Pública, División de Planificación Sanitaria, Departamento de Epidemiología Unidad de Estudios y Vigilancia de Enfermedades No Transmisibles. 2006 